\title{
Incubadoras en ambientes virtuales basadas en gestión de proyectos: Potenciando incubadoras de ciencia y emprendimiento, un aporte desde la academia*
}

Marvin Josué Aguilar Romero**, José Luis Rodríguez García**

\section{RESUMEN}

Para poder transmitir la cultura de la investigación y el emprendedurismo es necesario hacer partícipe a las personas interesadas en el desarrollo de iniciativas de emprendimiento y de proyectos de investigación científica, el modelo presentado a continuación plantea el uso de una incubadora virtual que, basándose en la gestión de proyectos, promete a los estudiantes obtener las competencias deseadas en emprendimiento y en investigación así como aumentar la producción científica de los países cuyas instituciones de educación superior hagan uso de las mismas.

Las salidas científicas alimentan a los proyectos de emprendimiento así como las diversas actividades de un emprendimiento alimentan a los proyectos de investigación para asegurar la calidad del producto final, así ambas incubadoras se complementan para duplicar la cantidad de salidas obtenidas y el enfoque modular brindado por la gestión de proyectos permite la integración multidisciplinaria en cada una de las tres fases del proceso de incubación: pre-incubación, incubación y postincubación.

Palabras clave: Incubadoras, ciencia, emprendimiento, producción científica.

\section{ABSTRACT}

In order to transmit the culture of research and entrepreneurship necessary to involve people in the development of entrepreneurial initiatives and scientific

Éste artículo surge como parte de la ponencia presentado en la décima Conferencia Internacional del Consorcio Latinoamericano y del Caribe de Escuelas de Ingeniería (por sus siglas en inglés LACCEI) y aparece en el proceeding del mismo ${ }^{1}$, por ello los autores han cambiado en la medida de lo posible el esquema del mismo enriqueciendo el contenido para ajustarlo a los requerimientos de ésta prestigiosa Revista; pero los lectores podrán encontrar cierta duplicidad de información con la que es propiedad de LACCEI.

** Universidad Nacional Autónoma de Honduras. Facultad de Ciencias Económicas. Instituto de Investigaciones Económicas y Sociales. maguilar@iies-unah.org 
research projects, the model presented below presents the use of a virtual incubator that based on management projects, promising students to obtain the desired competencies in entrepreneurship and research and increase the scientific output of countries with higher education institutions make use of them.

The scientific outputs feed the entrepreneurial projects and the various activities of a venture feed the research projects to ensure final product quality, and both incubators complement to double the amount of output obtained and the modular approach offered by the project management allows multidisciplinary integration in each of the three stages of incubation: pre-incubation, incubation and postincubation.

Keywords: Incubators, science, entrepreneurship, scientific production. 


\section{INTRODUCCIÓN}

Hablar de incubadoras no es algo novedoso e innovador, hay información sobre las mismas que data del año 1857 cuando en el Occidente Jean Louis Paul Denucé publica sobre las mismas ${ }^{2}$ ahora bien, "Urna de cristal en que se tiene a los niños nacidos antes de tiempo o en circunstancias anormales para facilitar el desarrollo de sus funciones orgánicas." (Real Academia Española) ${ }^{3}$ es la definición que podemos encontrar en el Diccionario de la Real Academia Española (DRAE) al buscar en ella el término de incubadora; Si tomamos como base el principio de las mismas y lo enmarcamos en un contexto general podríamos entonces referirnos a una incubadora como "El medio con ambientación controlada que asegura el desarrollo y crecimiento de quién, no teniendo las competencias propias, a ella se cobija mientras en ella se desarrollan los mecanismos para adquirir esas competencias y poder existir fuera de su cuidado" y si vamos más allá especificando un contexto para iniciativas de emprendedurismo podemos entonces pensar en incubar estas iniciativas para desarrollar productos, proyectos, empresas y demás productos que deriven de iniciativas de emprendimiento. Y quizá académicamente lo más importante, se pueden incubar investigaciones científicas.

El posicionamiento de las Universidades y mejora continua de la docencia depende en gran parte de la producción científica de estas instituciones, para ello es necesario contar no sólo con los centros destinados a este fin sino con el personal pertinente. La cultura de investigación se ha descuidado en América Latina y recientemente puede verse cómo la misma ha tomado relevancia y las Instituciones de Educación Superior (IES) comienzan a fomentar el desarrollo de esta cultura encontrándose con el hecho que, las personas capacitadas para estas funciones son pocas y es necesario transmitir estos conocimientos y experiencia a nuevas generaciones. El presente trabajo habla sobre una propuesta que pretende aumentar la producción de publicaciones científicas así como la inclusión y el acercamiento de docentes, especialistas, y de estudiantes a la investigación mediante el desarrollo metodológico de la misma.

\section{MÉTODOSYTÉCNICAS}

\subsection{Diseño:}

El trabajo presenta una investigación exploratoria sobre incubadoras y aborda el desarrollo de incubación basado en gestión de proyectos gestionada en un ambiente virtual mediante una herramienta de software. 


\subsection{Entorno:}

Al tratarse de un concepto nuevo, se indagó sobre incubadoras similares en centros de investigación de diferentes universidades, más que todo en américa latina, para lograr construir un modelo funcional y operativo de incubadoras.

\section{ANTECEDENTES EN INCUBACIÓN Y NACIMIENTO DE NUESTRA PROPUESTA}

\subsection{El proceso de incubación:}

Las primeras incubadoras ajenas al entorno biológico fueron las incubadoras de empresas en Silicon Valley, California en la década de los 50 por la Universidad de Stanford $^{4}$, desde esa fecha han sido innumerables la cantidad de incubadoras de empresas alrededor del mundo tanto que actualmente hay incubadoras que no se encuentran adjuntas a una IES.

Para el desarrollo de nuestras incubadoras utilizamos el mismo esquema usado por muchas incubadoras de empresas, cómo las del Tecnológico de Monterrey ${ }^{5}$, que dividen el proceso de incubación en tres etapas:

Pre-incubación: Es cuando los emprendedores se presentan con una idea y buscan desarrollar el plan de negocios para poder crear la empresa que gestione esta idea, en este punto reciben asesoría y se busca madurar la idea potenciándola con criterios que le permitan desarrollarse de una mejor manera. En esta etapa se presentan los análisis de mercadeo, se gestiona el plan estratégico (de negocios) e incluso es acá donde se constituye legalmente la empresa. Es muy importante porque en esta etapa se analizan los riesgos principales a los que puede ser vulnerable la nueva empresa y se tratan de minimizar.

Incubación: Se inicia el proceso mediante el cual la idea pasa de un plan estructurado de negocios a una empresa real, en este punto se vela por la seguridad económica de la empresa, el mercadeo, gestión de cuentas, etc... La incubadora puede brindar la infraestructura y mano de obra para los procesos asegurando un respaldo guiado y procesos de calidad. Acá obtenemos una empresa completamente operativa a la cual se pudo o no haber prestado espacio físico, infraestructura especial, maquinaria e incluso capital humano.

Post-incubación: Se da cuando la empresa abandona la incubadora para 
consolidarse y crecer, aunque depende menos de la incubadora siempre puede buscarle para asesoría hasta que ya no se requiera la comunicación. Cabe resaltar el hecho que, cada etapa difiere operacionalmente de la etapa anterior, pero necesitan realizarse por completo.

\subsection{Algunos tipos de incubadoras existentes:}

Como ya hemos mencionado anteriormente, las incubadoras de empresas fueron las primeras incubadoras ajenas a las concebidas originalmente; pero a través de los años muchas personas han utilizado las bondades de las mismas en diferentes ámbitos, lo que vino a enriquecer la producción en diversas áreas; entre las diferentes clases de incubadoras que se han manifestado es preciso para fines de nuestro trabajo mencionar las siguientes:

Incubadoras de Tecnología: Catalogadas en diferentes niveles (tecnología intermedia o de alta tecnología), la característica de estas incubadoras es el involucramiento de la innovación y de las TIC'S, por lo que se vinculan a centros e institutos de conocimiento, estas incubadoras no necesariamente incuban empresas sino que también proyectos que pueden ser bienes comercializables. Para mencionar algunas cifras, en México operan alrededor de 200 incubadoras tanto de empresas como de tecnología y en Israel encontramos unas 26 entre incubadoras de tecnología intermedia y alta.

Incubadoras en ambientes virtuales: Son aquellas en las cuales sus procesos se desarrollan asistidos por una plataforma virtual (generalmente vía intranet); La máxima de las mismas consiste en aprovechar el hecho que todos los procesos pueden realizarse asistidos por TIC's; pero en el caso especial de estas incubadoras, tenemos que las mismas gestionan los procesos de la incubación en una plataforma virtual (Castillo, 2007) ${ }^{6}$, un ejemplo de las mismas se encontraba como iniciativa en la Universidad Politécnica Nacional de México donde se plantea la gestión de una incubadora de empresas con apoyo de un ambiente virtual.

\subsection{Sobre las incubadoras propuestas en éste artículo:}

Para lograr aumentar la producción científica con materiales sensibles a propiedad intelectual de calidad académica y poder fomentar el emprendimiento es necesario la inclusión de estudiantes de IES y expertos en la práctica en un ambiente propicio para la investigación, de esta manera que los expertos puedan contar con apoyo en el desarrollo de la metodología y los estudiantes puedan aprender haciendo, de manos de expertos, el desarrollo del proceso, sumando estos principios a la 
experiencia en incubación encontrada en la investigación se propone el desarrollo de las siguientes incubadoras:

Incubadoras de Emprendimiento: Motivados por una iniciativa del director del Instituto de Investigaciones Económicas y Sociales de la Universidad Nacional Autónoma de Honduras (IIES-UNAH), se pensó en idear un mecanismo de desarrollar de manera metódica iniciativas de emprendimiento que no fueran empresas, las mismas en su fase final no proporcionarían un patrimonio sino un producto completo.

La incubadora de emprendimiento es una incubadora que vincula a la incubadora de empresas con las incubadoras de tecnología (intermedia o alta) en un ambiente virtual, en esta incubadora se puede incubar, no sólo empresas sino que proyectos o cualquier actividad que tenga como resultado una aplicación práctica. Tiene la ventaja que tratará las iniciativas de proyectos como a empresas y en todo el proceso del desarrollo se le cuidará y potenciará hasta obtener el producto deseado pudiendo además ser usada como una incubadora de empresas virtual convencional.

Incubadoras de Ciencia: Motivados por las capacidades que prestan las incubadoras encontradas y las necesidades de contar con calidad académica en los procesos se concibió la idea de una incubadora que pudiese potenciar la investigación al gestionar su metodología generando así una incubadora de ciencias. Cumpliendo con la disciplina de investigación se propone una herramienta que permita forjar investigadores y obtener conocimiento de calidad científica y académica que pueda utilizarse para el desarrollo de la educación en todos sus niveles, en especial el nivel superior. Estas incubadoras operarán de manera similar a una empresa de alta tecnología con las tres partes del proceso de incubadoras convencionales de la siguiente manera:

Pre-incubación: Acá se analizará la viabilidad del proyecto (ya sea de investigación o de emprendimiento), se delimitarán los alcances y se clasificarán las ideas para asegurar el desarrollo y la producción de resultados.

Incubación: Si se habla de un proyecto científico la metodología de la investigación se pondrá en práctica, los involucrados podrán iniciarse en el campo de la investigación bajo la tutela de personas con experiencia en la materia; mientras que, si se refiere a una idea de emprendimiento los procesos de gestión de una incubadora convencional se pueden adaptar para el desarrollo del producto que se desee lograr. 
Post-incubación: De cada proyecto de investigación se obtendrán numerosas salidas, la mayoría sensible a la propiedad intelectual, y en esta parte del proceso se socializarán resultados y se incluirá en la academia el conocimiento debido; mientras que de cada incubación de emprendimiento se obtendrán planos, diseños, prototipos, modelos, productos sensibles a la propiedad intelectual además de un producto funcional.

\subsection{Hacer operativas a la incubadora de ciencia y a la incubadora de emprendimiento}

Como se habrá podido vislumbrar ambas iniciativas difieren de las empresas (que es lo que se acostumbra a incubar); pero eso no va en contra del proceso de incubación, por el contrario es necesario asegurar que dicho proceso facilite el desarrollo de la iniciativa y asegure la obtención de salidas a la vez que incorpora capital humano para la transferencia de la experiencia y el desarrollo de la metodología. En el caso de iniciativas de investigación puede usarse una metodología que dependa del tipo de investigación para lograr finalizarle y, en el caso de una de emprendimiento se puede dividir su desarrollo en módulos y etapas.

Como uno de los objetivos de la incubadora es precisamente fomentar la investigación y hacer partícipes a los estudiantes de la misma (que se ha mencionado varias veces), es necesario contar con expertos que puedan guiar a quienes incursionan en el proceso sin interferir con el desarrollo de la iniciativa que se está incubando, por ello las incubadoras (la de ciencia y la de emprendimiento) gestionan sus iniciativas como proyectos, cada iniciativa se divide modularmente en actividades con un alcance determinado (inicio, final), un tiempo de ejecución (dictado en primera instancia por los especialistas y triangulado con los requeridos en incubaciones anteriores), un presupuesto (que puede provenir de quienes generan la iniciativa o de patrocinadores, así como de fondos propios de las incubadoras) y una adecuada gestión para desarrollarse así, cada actividad puede sub-dividirse a su vez en tareas e indicar uno o más responsables en la ejecución de las mismas. Los estudiantes que participen en la incubación de una iniciativa serán quienes, guiados por expertos en la materia, desarrollaran las tareas y presentarán los resultados de las mismas, el conjunto de resultados obtenidos en cada tarea son los insumos del resultado de la actividad de esta manera puede evaluarse las tareas, las actividades, a quienes ejecutan las tareas y la asistencia brindada por los expertos en el proceso sin comprometer el desarrollo de la incubación y serán los encargados de la incubación quienes velen por el desarrollo de las etapas de todo el proceso. 
Por todo lo anterior, que facilita el proceso de gestión, se decidió basar el desarrollo de las etapas de incubación en la gestión de proyectos.

Para hacer operativa la incubadora serán necesarios los siguientes actores:

- Estudiantes que deseen participar en la incubación, estos serán quienes desarrollen las tareas contenidas en las actividades y la selección de los mismos dependerá de las necesidades de cada iniciativa de emprendimiento.

- Docentes especialistas en el área de la iniciativa de emprendimiento, se convertirán en consultores que ayudarán a construir las actividades, definir la cantidad de estudiantes que deben participar en cada una, colaborarán asesorando a los estudiantes en el desarrollo de las tareas y revisarán los resultados.

- Un ente que administre la incubadora virtual, encargados del control de consultores, emprendedores, investigadores, comunicación entre estudiantes y consultores, y gestión de la incubación.

- Un ente gestor que vele porque quién administre la incubadora lo haga de la mejor manera posible.

- Fuente de financiamiento cuando se requiera.

En el diagrama 1 puede apreciarse un bosquejo de la relación básica de actores en el proceso de incubación.

Diagrama: Relación básica de principales actores en el proceso de incubación basado en gestor de proyectos, construcción propia

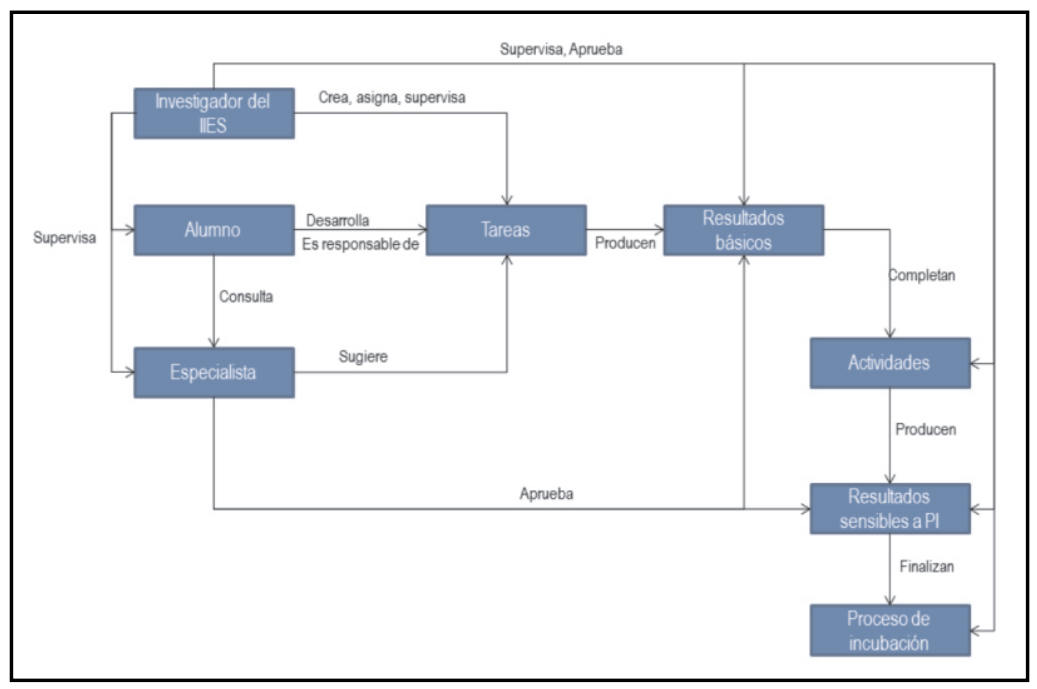




\section{CONCLUSIONES}

A lo largo del documento se ha expuesto la creación y cómo hacer operativo un sistema de información que, ejecutándose en un ambiente virtual, permita mediante la gestión de proyectos el desarrollo de la ciencia y el emprendimiento; Es la oportunidad de poder transferir las competencias de investigación y emprendimiento de expertos a estudiantes sin comprometer de manera alguna una iniciativa ni frenar la producción científica.

La potencialidad de esta herramienta se aumenta al establecerse que, la salida que provee una incubadora puede alimentar a la otra como insumo y es posible que durante el proceso de incubación, una actividad requiera para completarse una nueva iniciativa de incubación en sí misma o en su hermana así ambas incubadoras pueden alimentarse entre ellas y ambas pueden beneficiar a la sociedad en general sin comprometer el desarrollo de las iniciativas, y desarrollando salidas que eleven el nivel de las instituciones de educación superior y provean de productos de calidad.

El IIES-UNAH desde el 2010 ha concebido, diseñado, desarrollando y está por poner en marcha ambas incubadoras innovando en la educación superior.

\section{AGRADECIMIENTOS}

Los autores desean agradecer a la Dirección de Investigación Científica de la Universidad Nacional Autónoma de Honduras por la oportunidad de publicar en su revista, así como por el financiamiento requerido para la presentación de la ponencia en el décimo congreso de LACCEI y, agradecemos también a los investigadores Msc. Manuel Flores Fonseca y al Lic. Nelson Roberto Raudales por su valiosa colaboración e instrucción para la redacción y construcción de textos científicos, sin sus observaciones este artículo no hubiese visto la luz.

\section{BIBLIOGRAFÍA}

1. Proceeding de la $10^{\mathrm{a}}$ Conferencia Internacional del Consorcio Latinoamericano y del Caribe de Escuelas de Ingeniería. http://www.laccei.org/index.php/publications/laccei-proceedings

2. Morilla Guzmán, Andrés. (@ 1999-2012 - Centro Nacional de Información de Ciencias Médicas). Historia de la incubadora, 1-6. Recuperado el 20 de Junio de 2012, de 
http://www.sld.cu/galerias/pdf/sitios/pediatria/historia_de_la_incubadora.pdf

3. Real Academia de la lengua española, (Real Academia Española ( )). Incubadora. Recuperado el 12/10/2010 en http://lema.rae.es/drae/?val=incubadora

4. Toledo, Cristian. (25 de septiembre de 2007). Historia de Incubadoras de Empresas. Recuperado el 14/10/2010 en http://incubacionempresas.wordpress.com/2007/09/25/hola-mundo/

5. Incubadora de empresas, Tecnológico de monterrey. http://incubadoras.mty.itesm.mx/modelo.php, 11/20/2010.

6. Castillo Martha, "Incubación de empresas en Ambientes Virtuales". IPN México http://www.ciie.cfie.ipn.mx/2domemorias/documents/m/m13b/m13b_48.pdf, 04/20/2012. 